\title{
Staudinger Ligation of Peptides at Non-Glycyl Residues
}

\author{
Matthew B. Soellner, ${ }^{\dagger, \dagger}$ Annie Tam, ${ }^{\dagger}$ and Ronald T. Raines ${ }^{*}, \dagger, \S$ \\ Departments of Chemistry and Biochemistry, \\ University of Wisconsin-Madison, Madison, WI 53706
}

\begin{tabular}{|c|c|}
\hline Page & Contents \\
\hline S1 & Table of Contents \\
\hline $\mathrm{S} 2$ & General Experimental Procedures \\
\hline $\mathrm{S} 3$ & ${ }^{1} \mathrm{H}$ NMR Spectrum of Compound $\mathbf{1 0}$ \\
\hline $\mathrm{S} 4$ & ${ }^{13} \mathrm{C}$ NMR Spectrum of Compound $\mathbf{1 0}$ \\
\hline S5 & ${ }^{31} \mathrm{P}$ NMR Spectrum of Compound $\mathbf{1 0}$ \\
\hline S6 & ${ }^{1} \mathrm{H}$ NMR Spectrum of Compound 14 \\
\hline S7 & ${ }^{13} \mathrm{C}$ NMR Spectrum of Compound 14 \\
\hline S8 & ${ }^{31} \mathrm{P}$ NMR Spectrum of Compound $\mathbf{1 4}$ \\
\hline S9 & ${ }^{1} \mathrm{H}$ NMR Spectrum of Compound 15 \\
\hline S10 & ${ }^{13} \mathrm{C}$ NMR Spectrum of Compound $\mathbf{1 5}$ \\
\hline $\mathrm{S} 11$ & ${ }^{31} \mathrm{P}$ NMR Spectrum of Compound $\mathbf{1 5}$ \\
\hline $\mathrm{S} 12$ & ${ }^{1} \mathrm{H}$ NMR Spectrum of Compound 18 \\
\hline S13 & ${ }^{13} \mathrm{C}$ NMR Spectrum of Compound $\mathbf{1 8}$ \\
\hline S14 & ${ }^{31} \mathrm{P}$ NMR Spectrum of Compound $\mathbf{1 8}$ \\
\hline S15 & ${ }^{1} \mathrm{H}$ NMR Spectrum of Compound $\mathbf{2 1}$ \\
\hline S16 & ${ }^{13} \mathrm{C}$ NMR Spectrum of Compound $\mathbf{2 1}$ \\
\hline S17 & ${ }^{31} \mathrm{P}$ NMR Spectrum of Compound $\mathbf{2 1}$ \\
\hline S18 & ${ }^{1} \mathrm{H}$ NMR Spectrum of Compound $\mathbf{2 4}$ \\
\hline S19 & ${ }^{13} \mathrm{C}$ NMR Spectrum of Compound 24 \\
\hline S20 & ${ }^{31} \mathrm{P}$ NMR Spectrum of Compound $\mathbf{2 4}$ \\
\hline $\mathrm{S} 21$ & ${ }^{1} \mathrm{H}$ NMR Spectrum of Compound 25 \\
\hline S22 & ${ }^{13} \mathrm{C}$ NMR Spectrum of Compound 25 \\
\hline S23 & ${ }^{31} \mathrm{P}$ NMR Spectrum of Compound 25 \\
\hline S24 & ${ }^{1} \mathrm{H}$ NMR Spectrum of Compound $\mathbf{2 6}$ \\
\hline $\mathrm{S} 25$ & ${ }^{13} \mathrm{C}$ NMR Spectrum of Compound $\mathbf{2 6}$ \\
\hline S26-28 & Data from Theoretical Calculations for Table 1 \\
\hline S29-30 & Data from Theoretical Calculations for Table 2 \\
\hline
\end{tabular}




\section{General Experimental Procedures}

General Materials. Reagent chemicals were obtained from commercial suppliers, and reagent-grade solvents were used without further purification. Reactions were performed at room temperature, and were monitored by thin-layer chromatography with visualization by UV light or staining with ninhydrin or $\mathrm{I}_{2}$. Compounds were purified with an Argonaut Flashmaster Solo automated chromatography system unless indicated otherwise. Silica gel used in flash chromatography had 230-400 mesh and 60 - $A$ pore size.

General Methods. The removal of solvent "under reduced pressure" refers to the use of a rotary evaporator at water aspirator pressure $(<20$ torr $)$ while maintaining the water-bath temperature below $40^{\circ} \mathrm{C}$. Residual DMF was removed from samples at high vacuum ( $<0.1$ torr) by the use of a mechanical belt-drive oil pump.

NMR Spectroscopy and Mass Spectrometry. NMR spectra were obtained with a 500 or $400 \mathrm{MHz}$ spectrometer at the National Magnetic Resonance Facility at Madison or the University of Wisconsin Nuclear Magnetic Resonance Facility, respectively. Carbon-13 and phosphorus-31 spectra were both proton-decoupled, and phosphorus-31 spectra were referenced against an external standard of deuterated phosphoric acid $(0 \mathrm{ppm})$. Mass spectra were obtained with electrospray ionization (ESI) techniques.

General Procedures for Staudinger Ligations. Unless noted otherwise, Staudinger ligations were performed at room temperature with equimolar amounts of phosphinothioester (or phosphinoester) and azide $(0.105 \mathrm{mmol})$ in a solvent $(600 \mu \mathrm{L})$. Solvents were not degassed, and ligations were performed under air. 


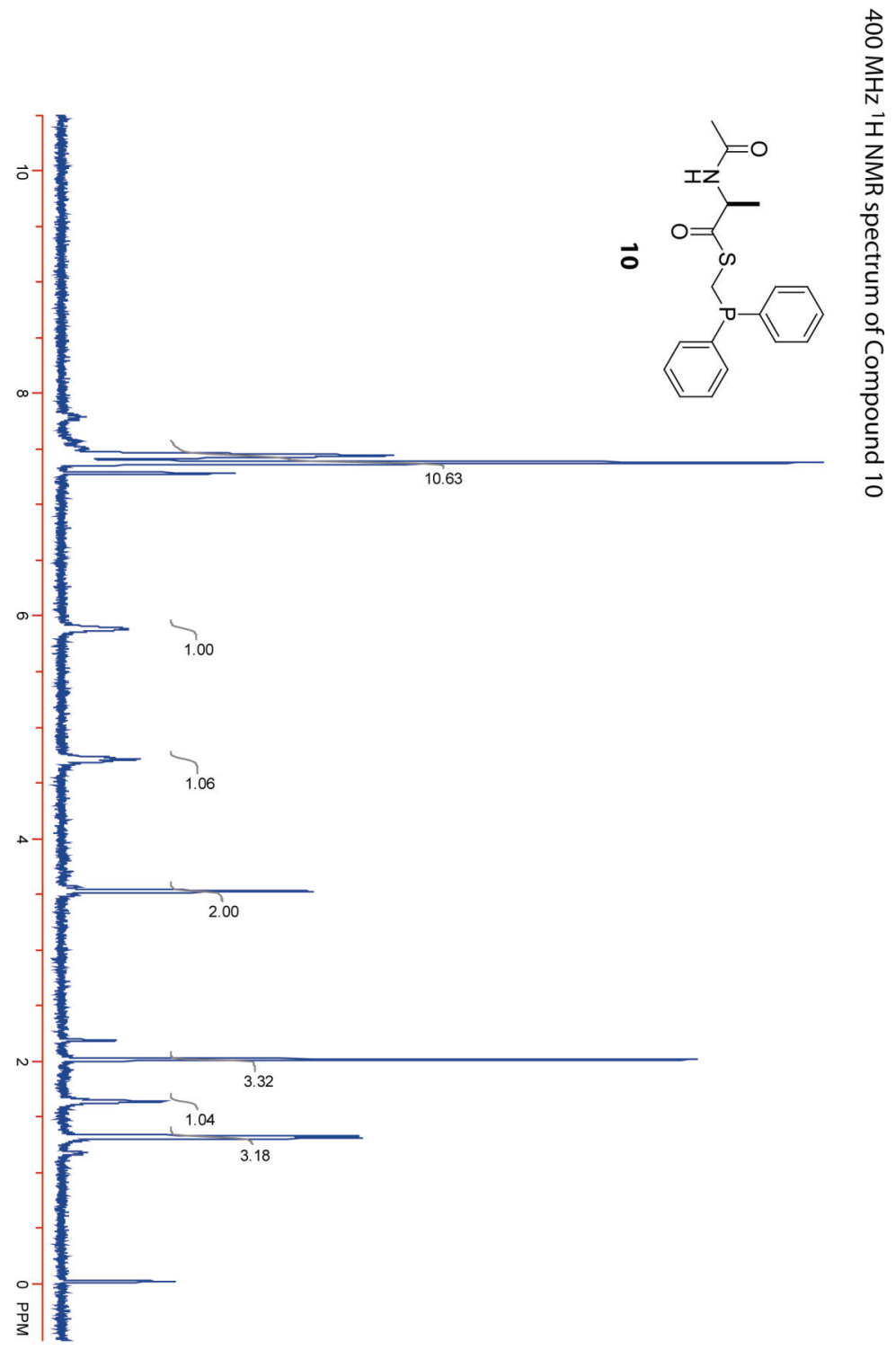




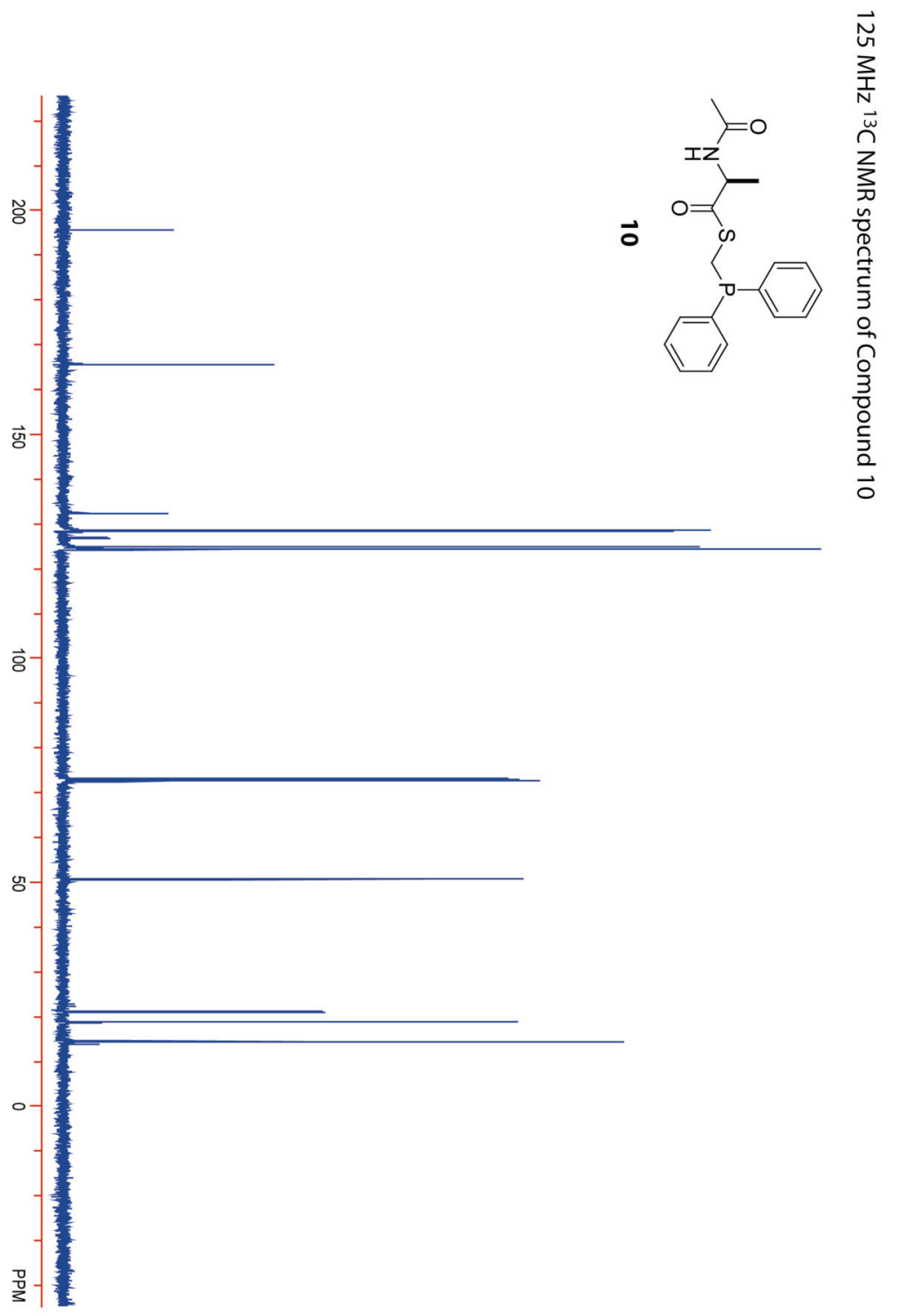



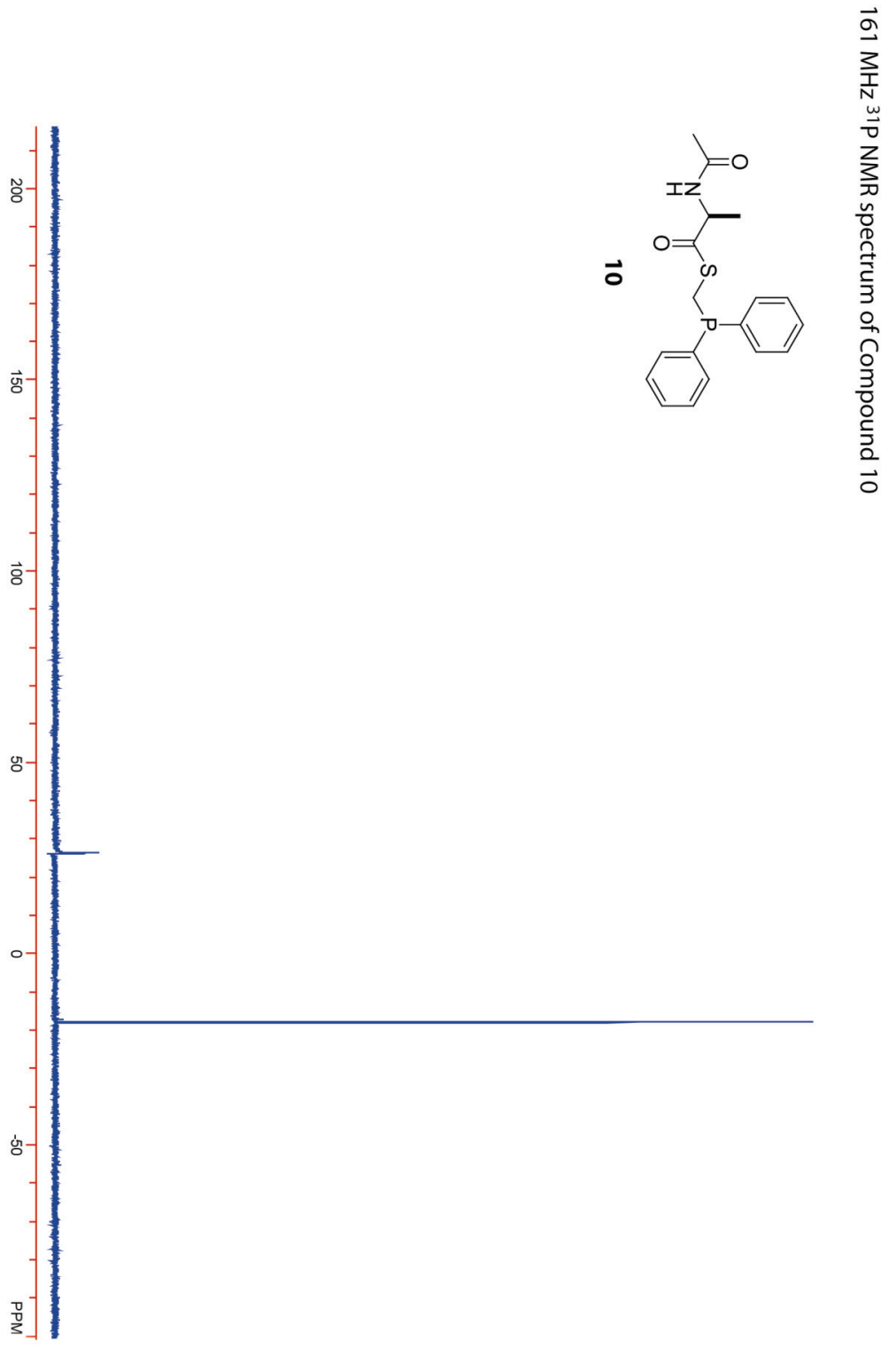


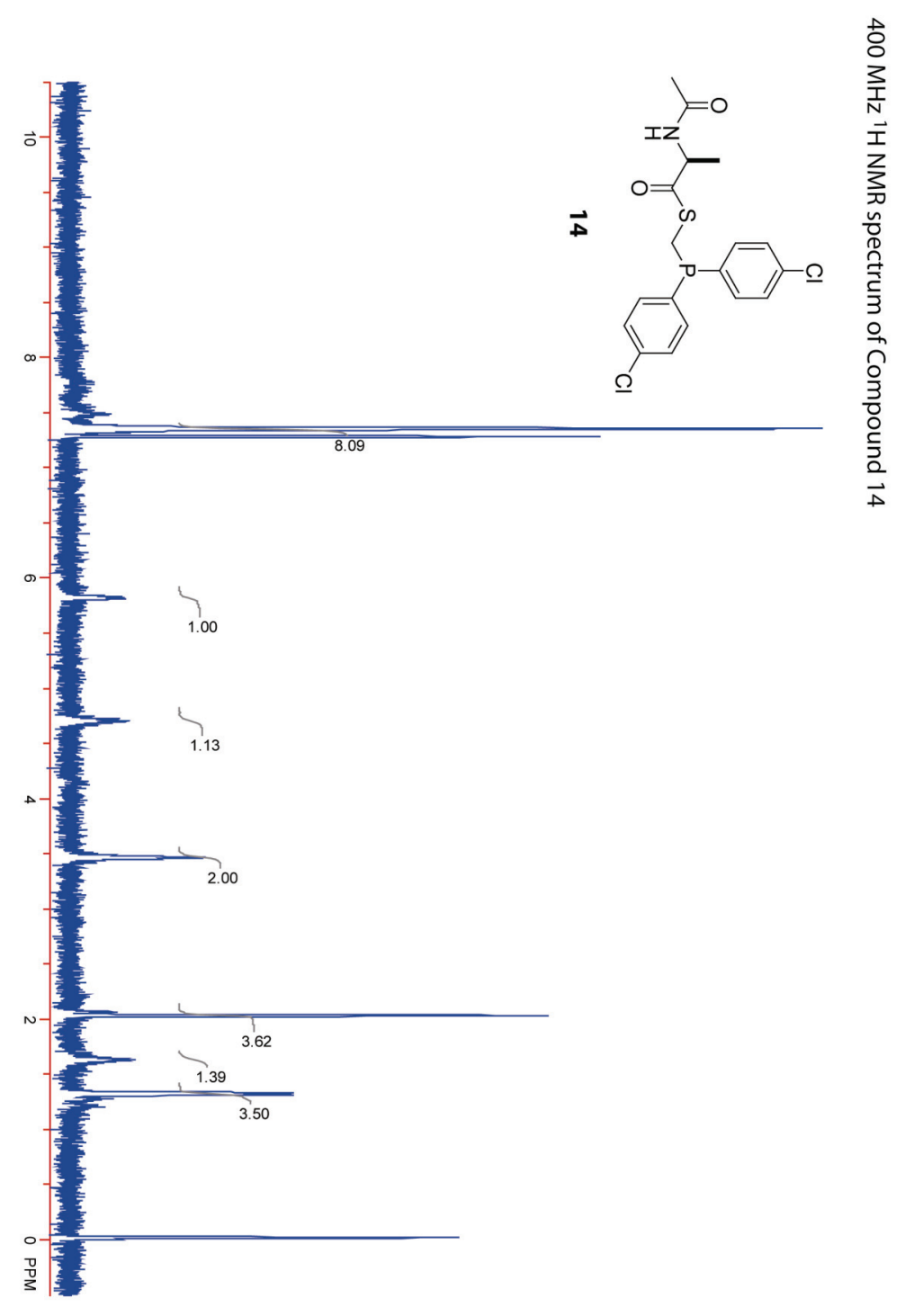




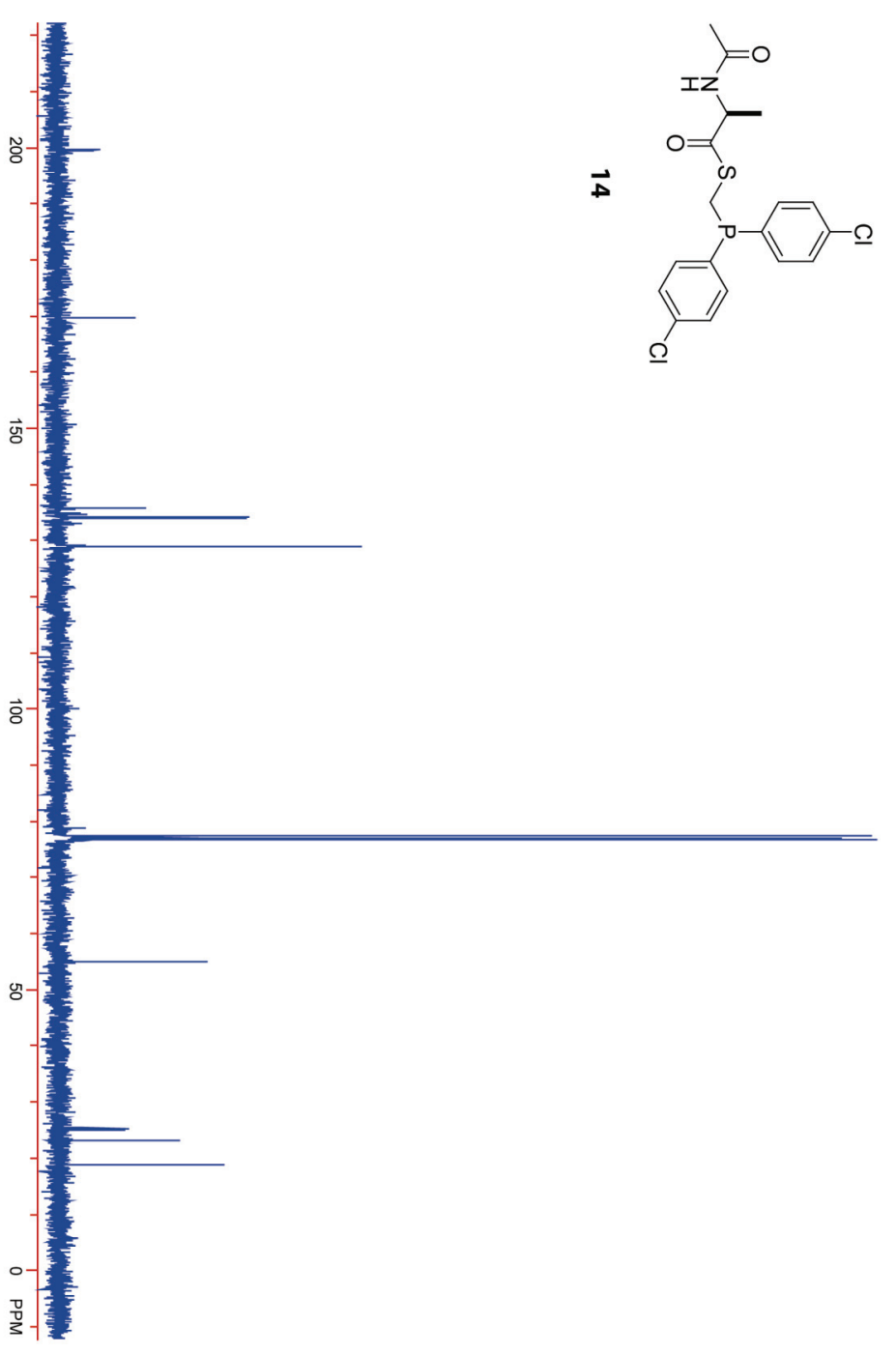

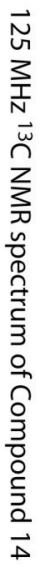



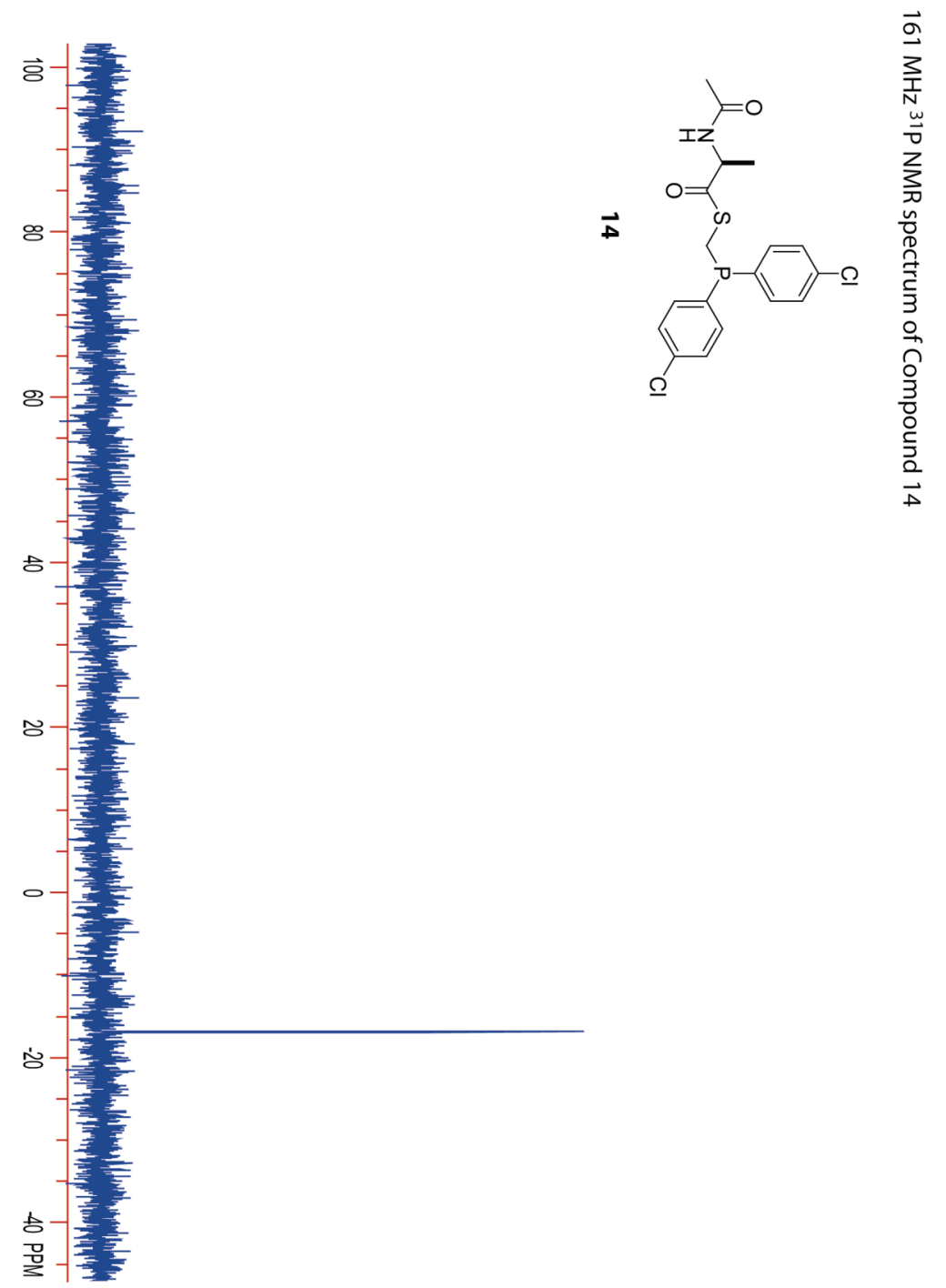


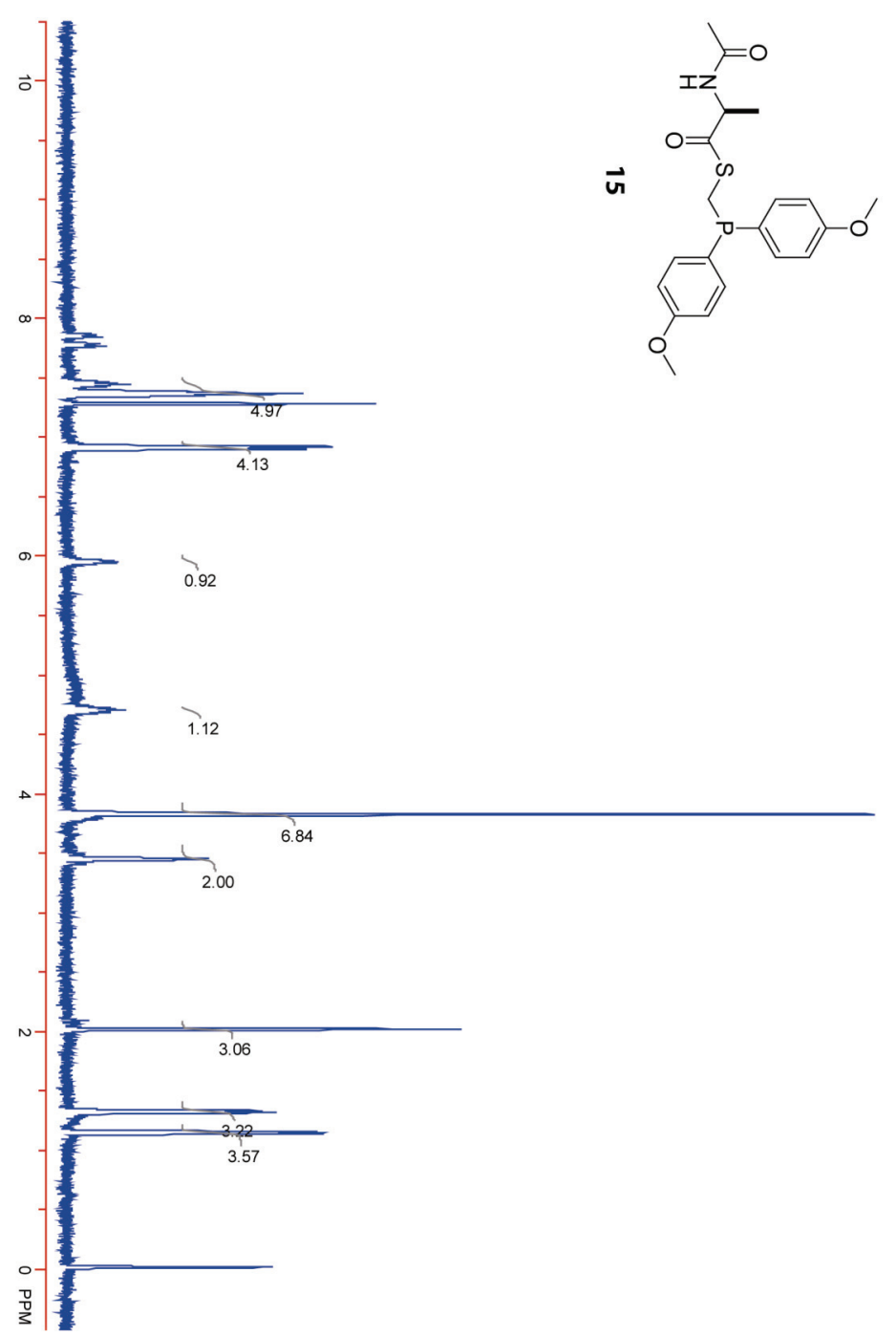

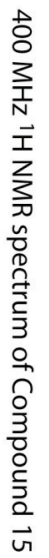




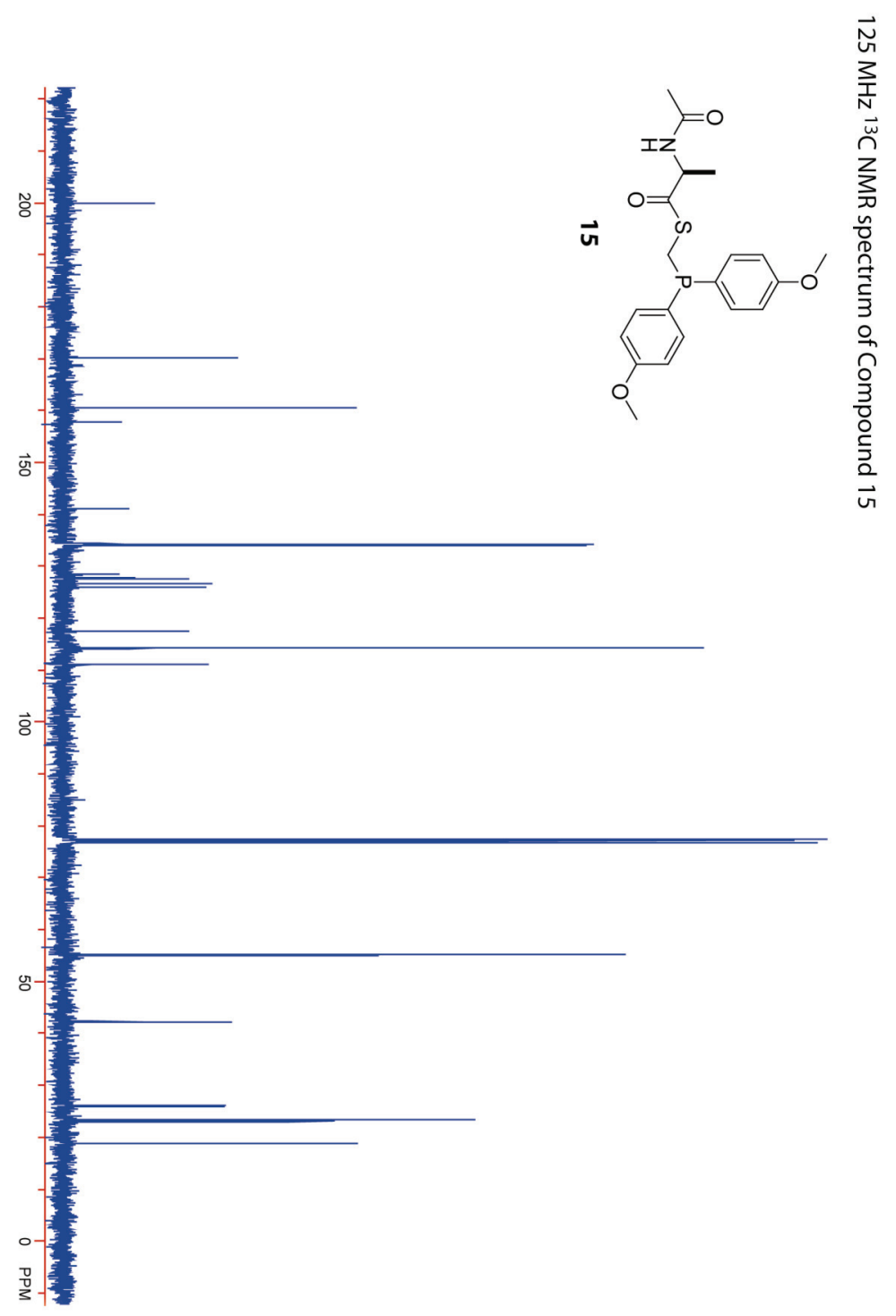




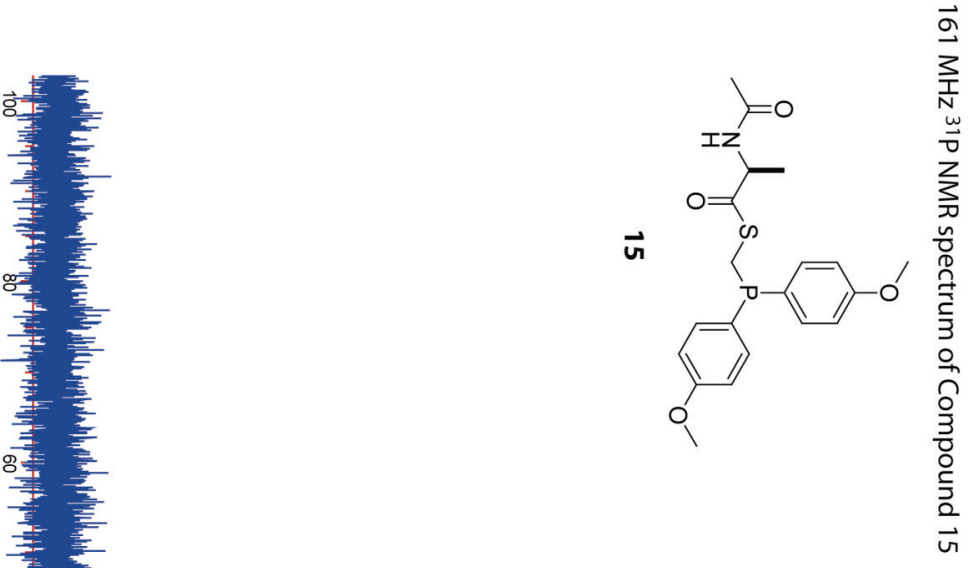




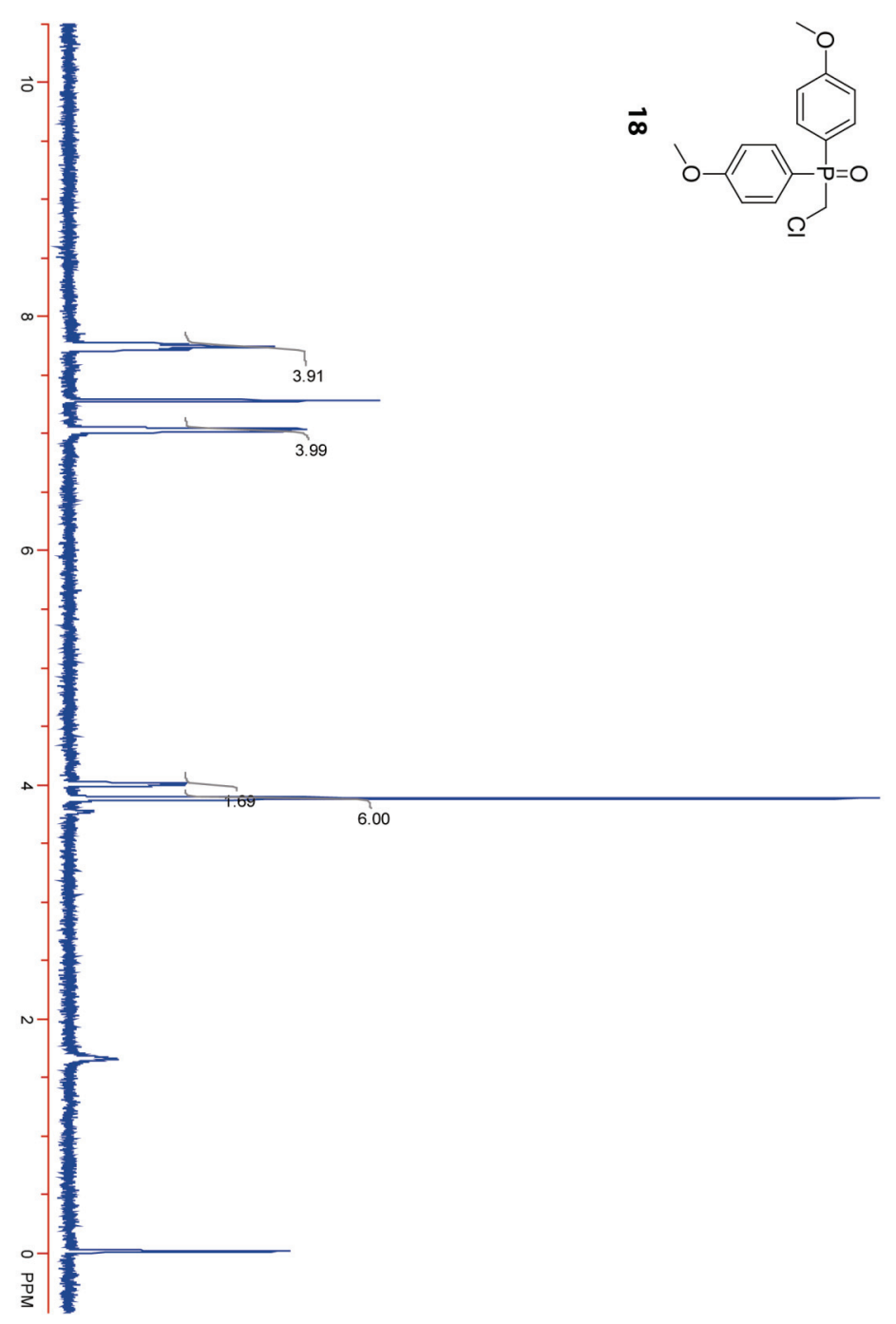




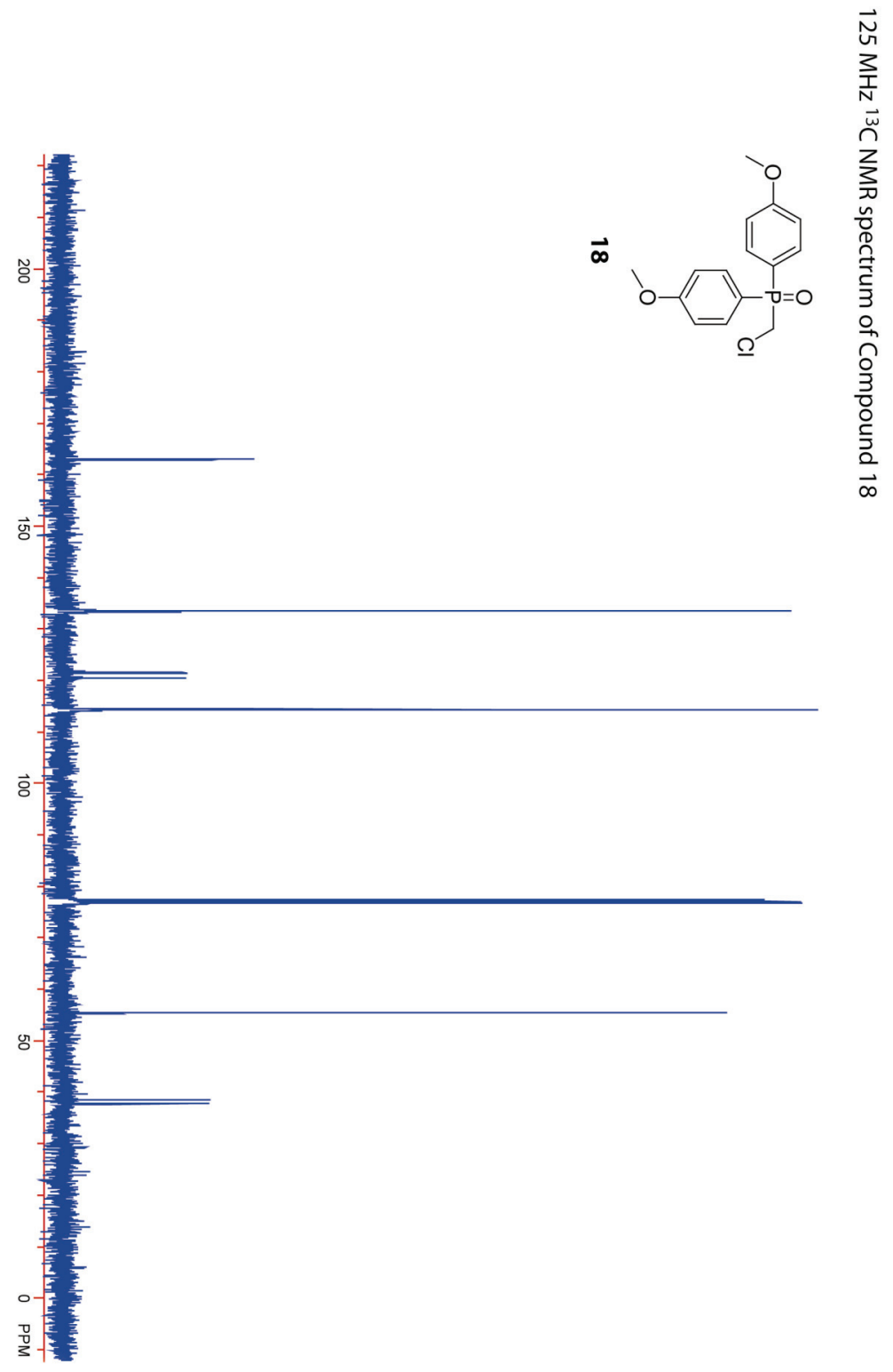




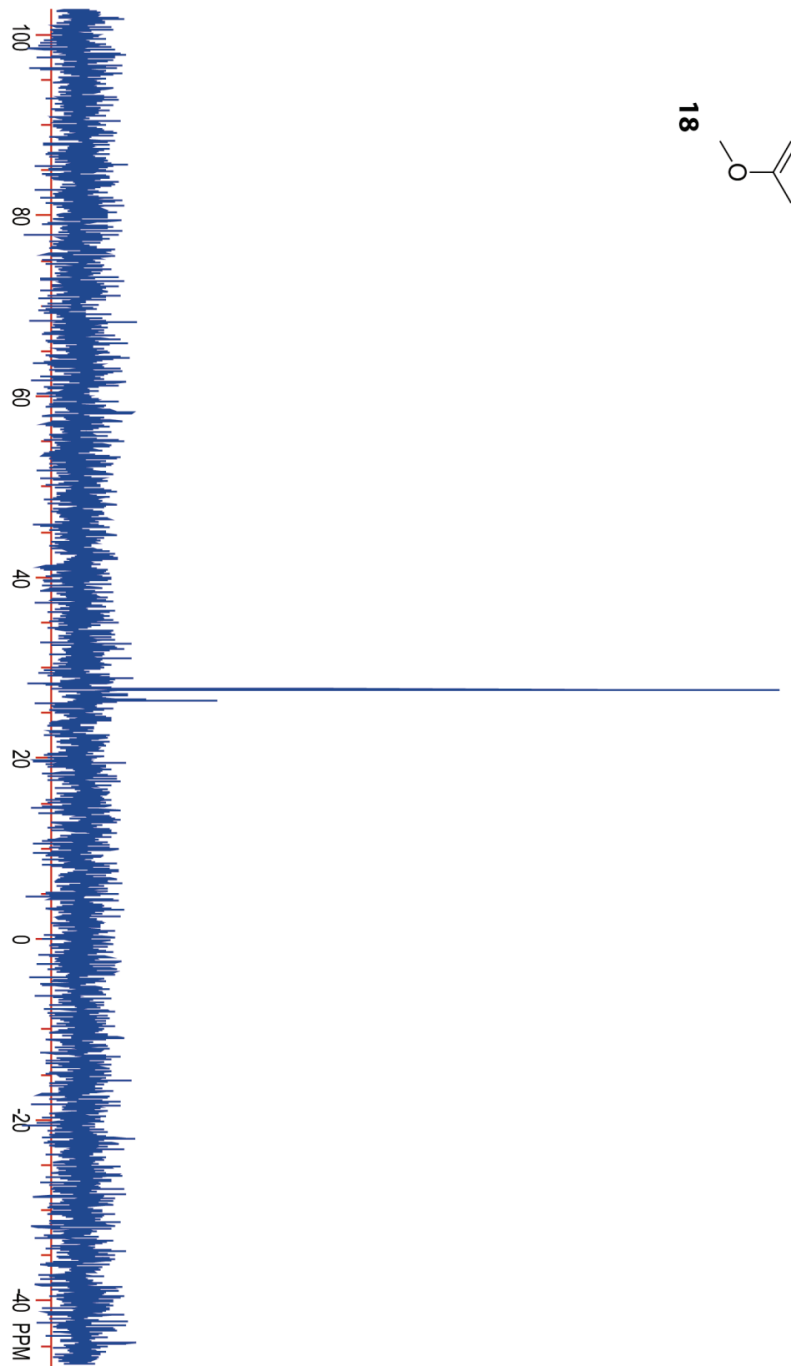




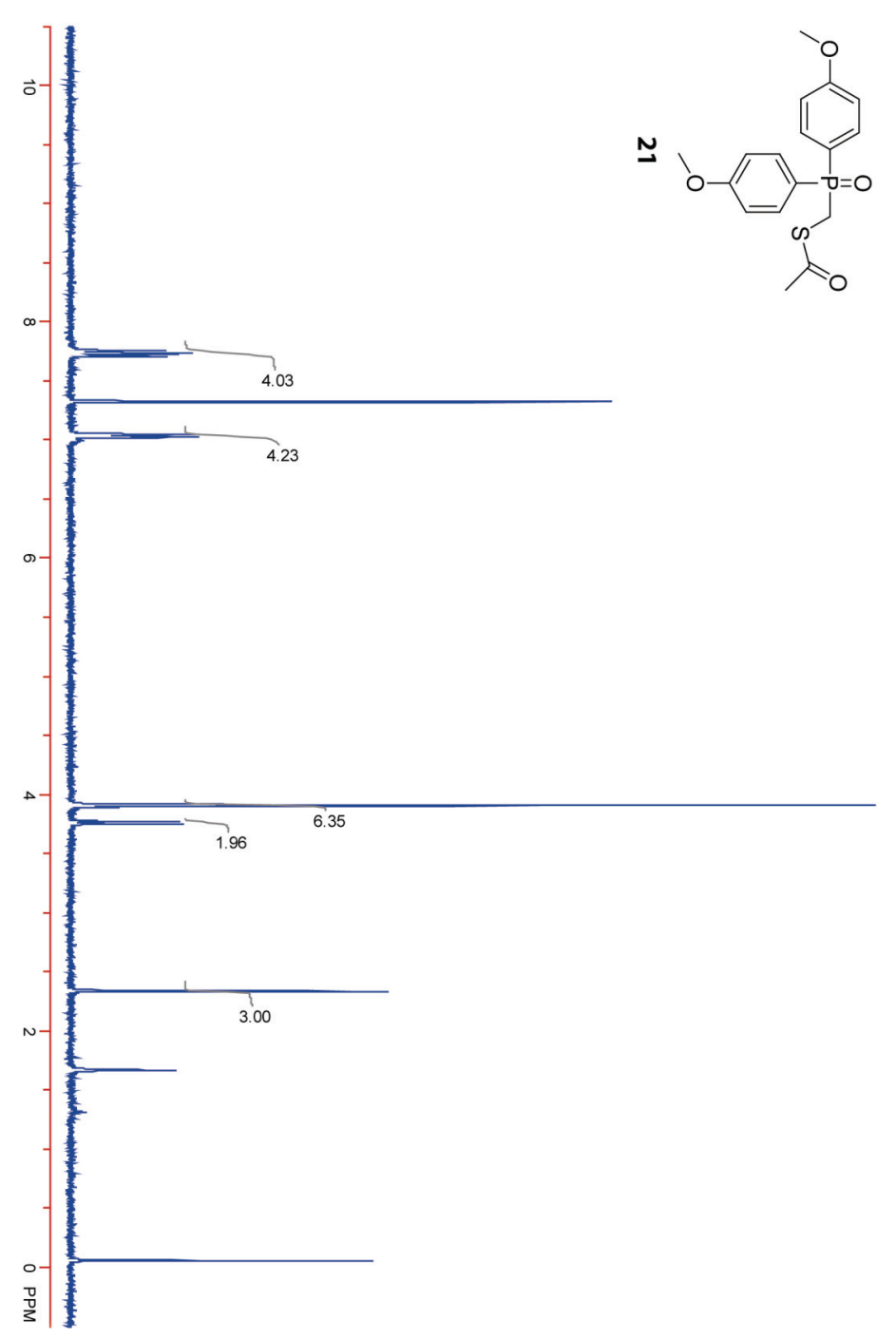

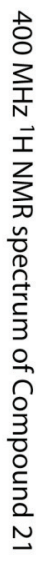



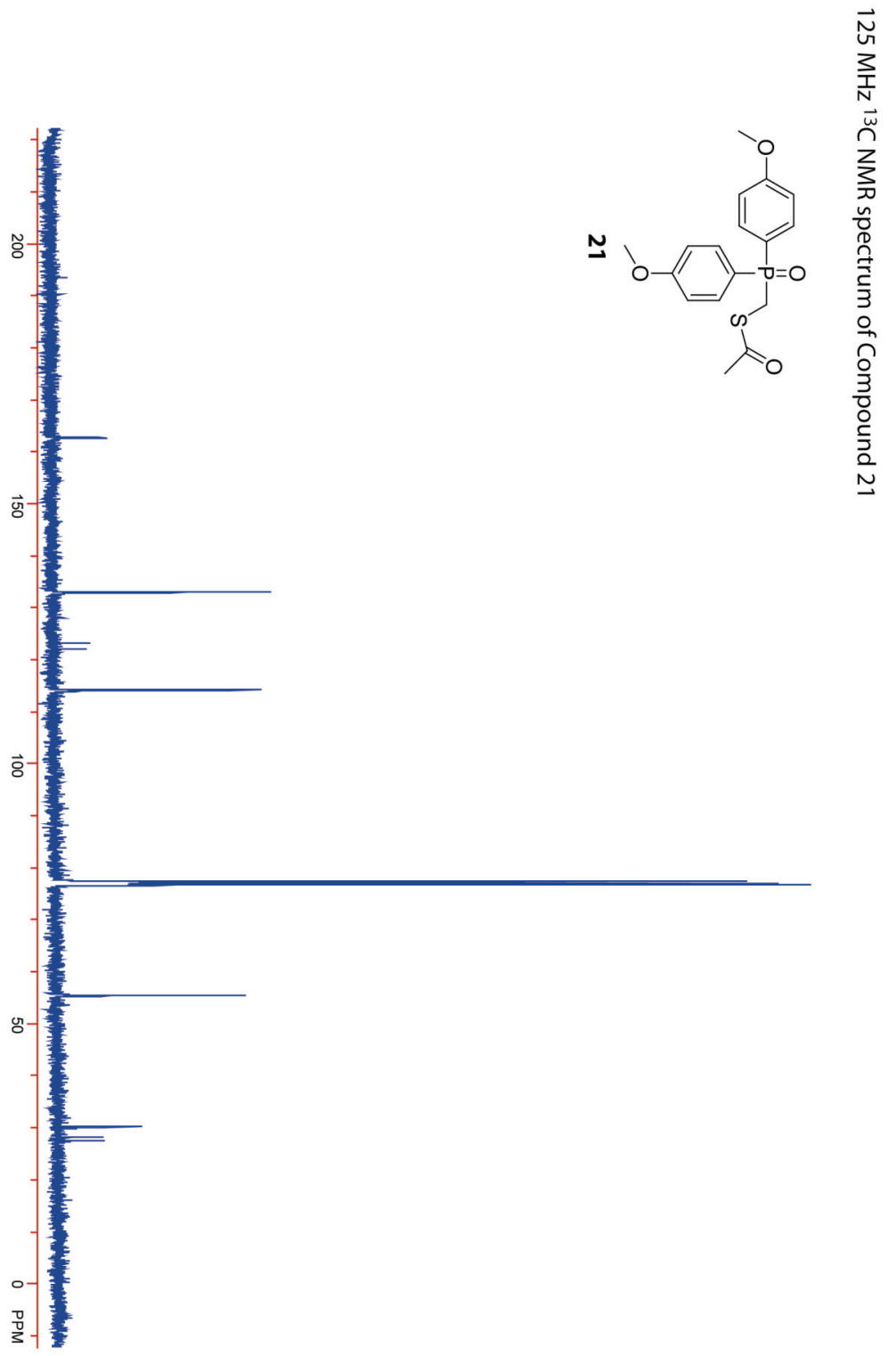

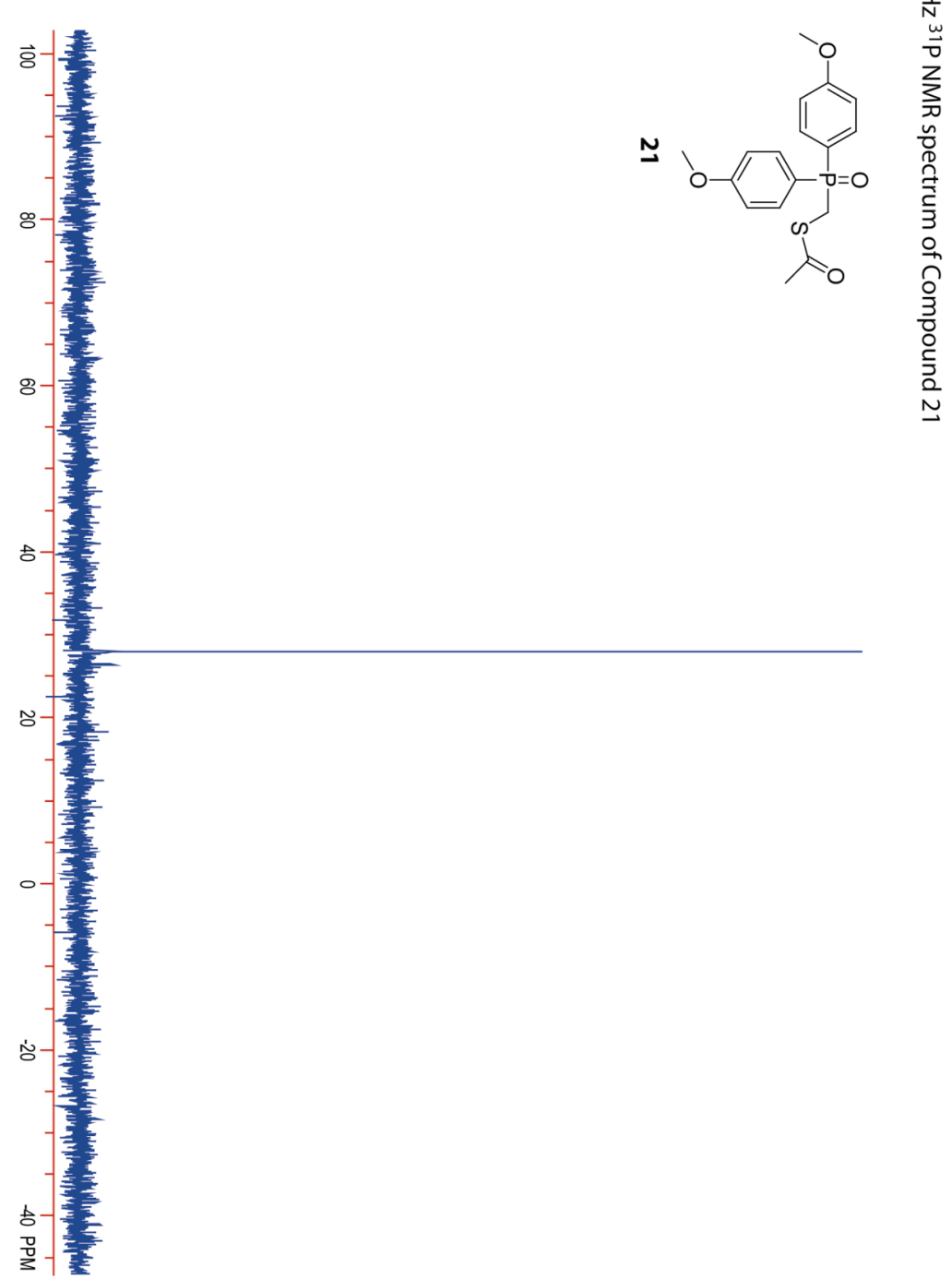


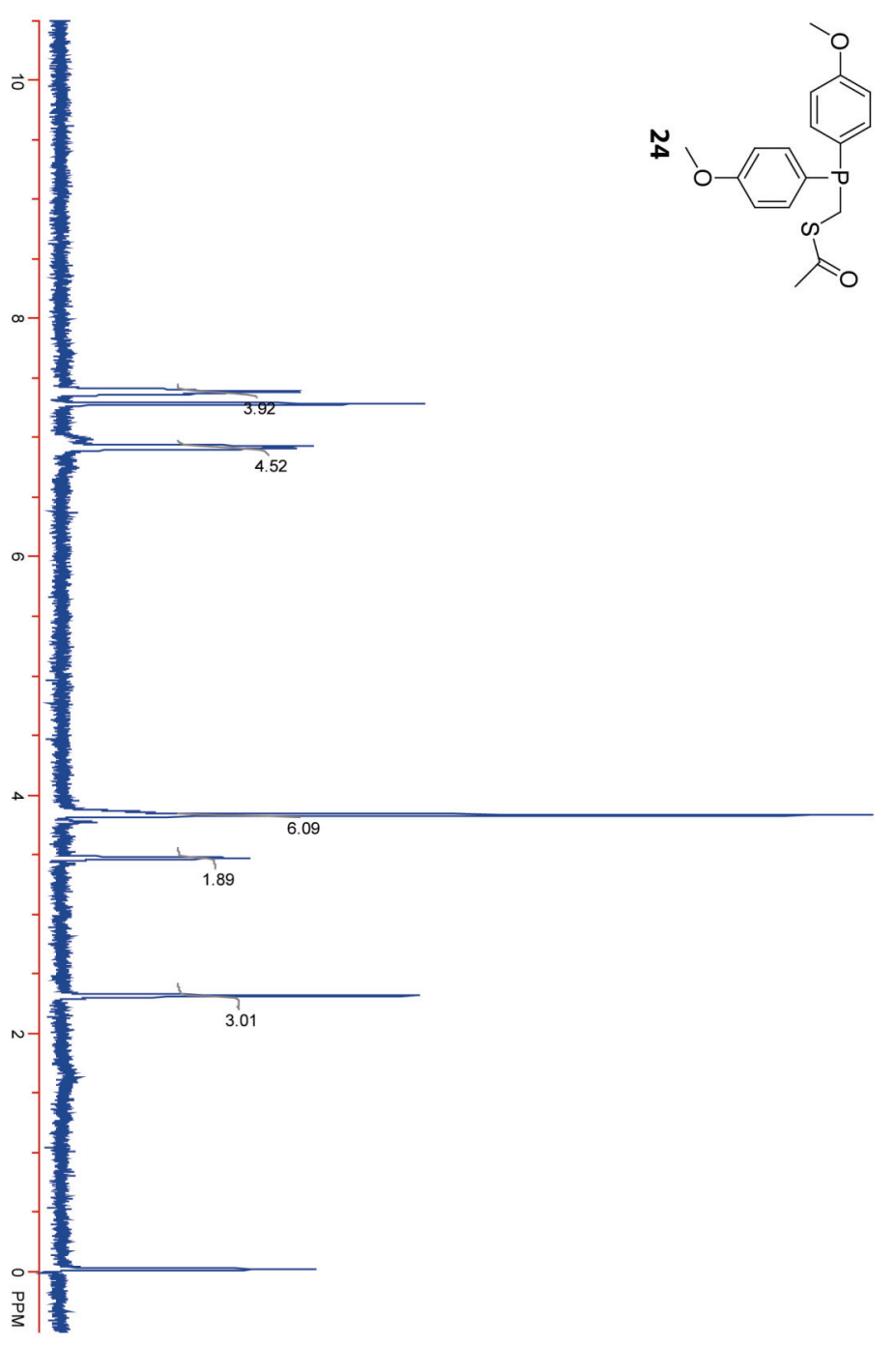

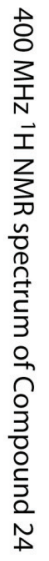




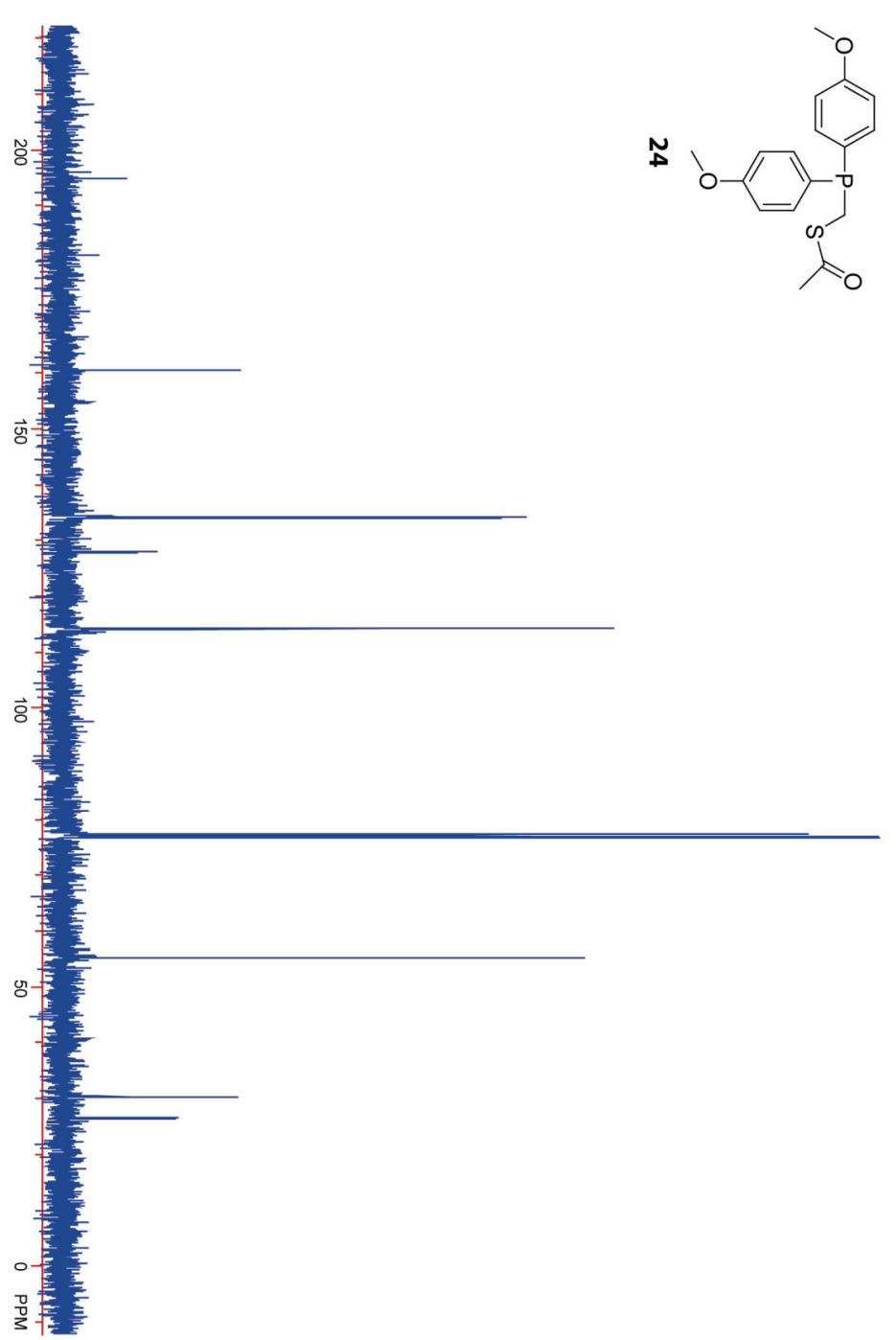

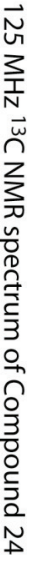




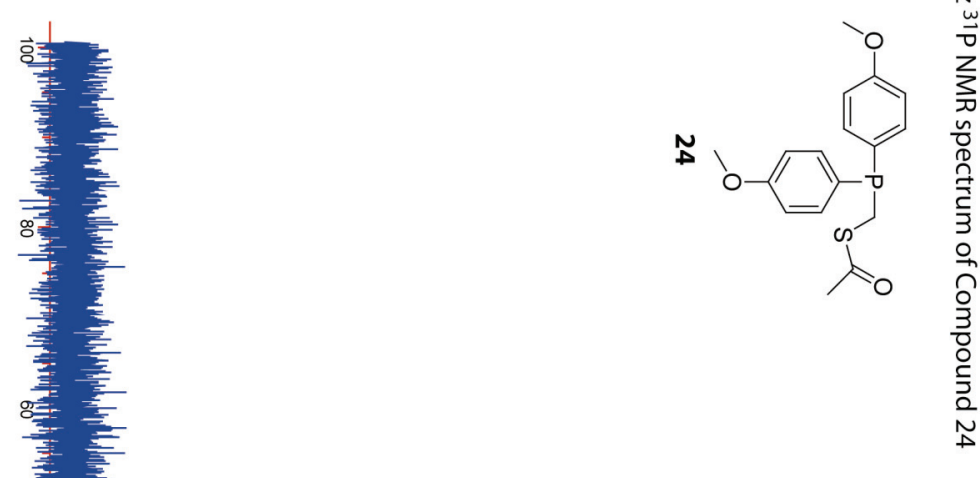




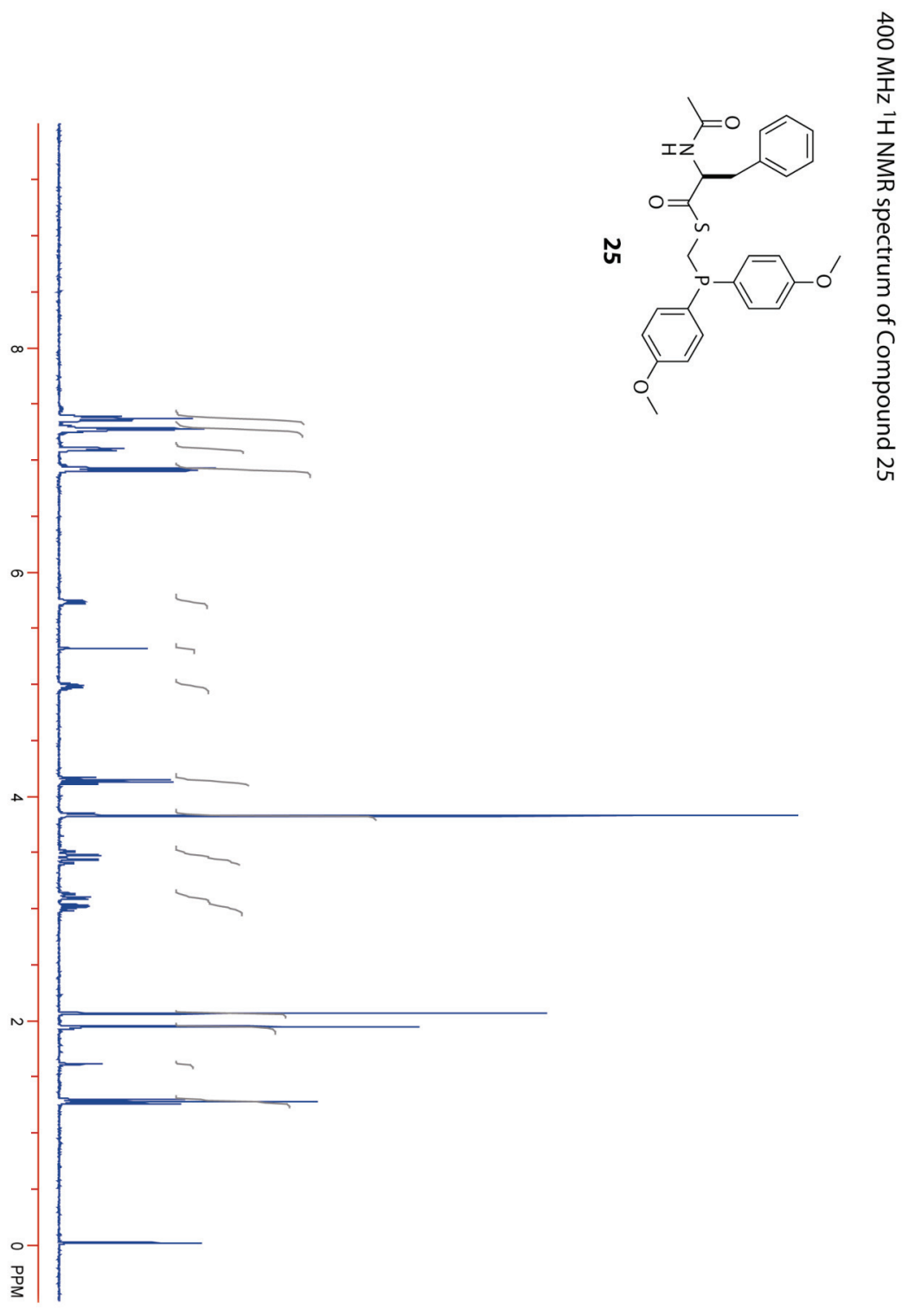




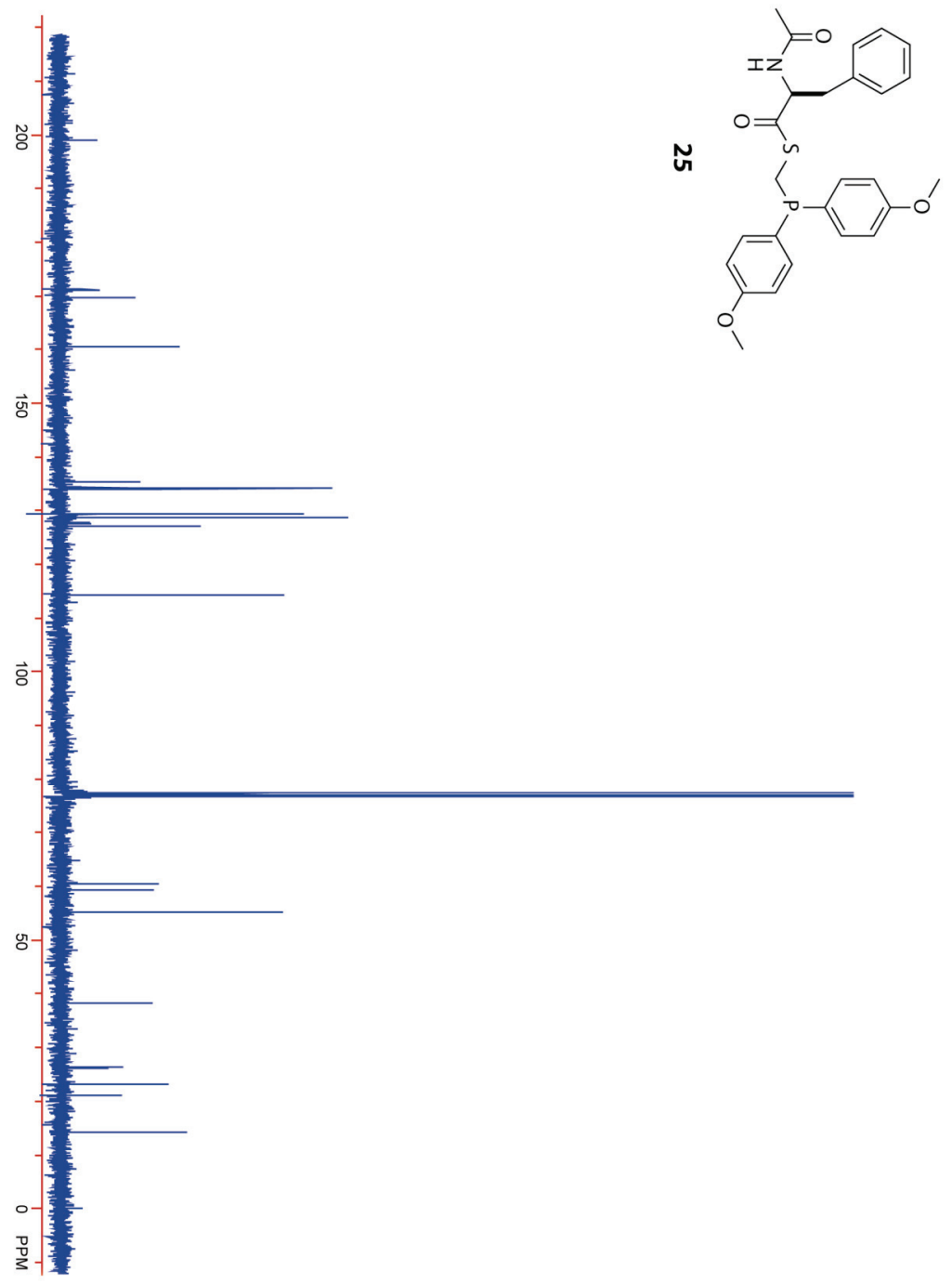



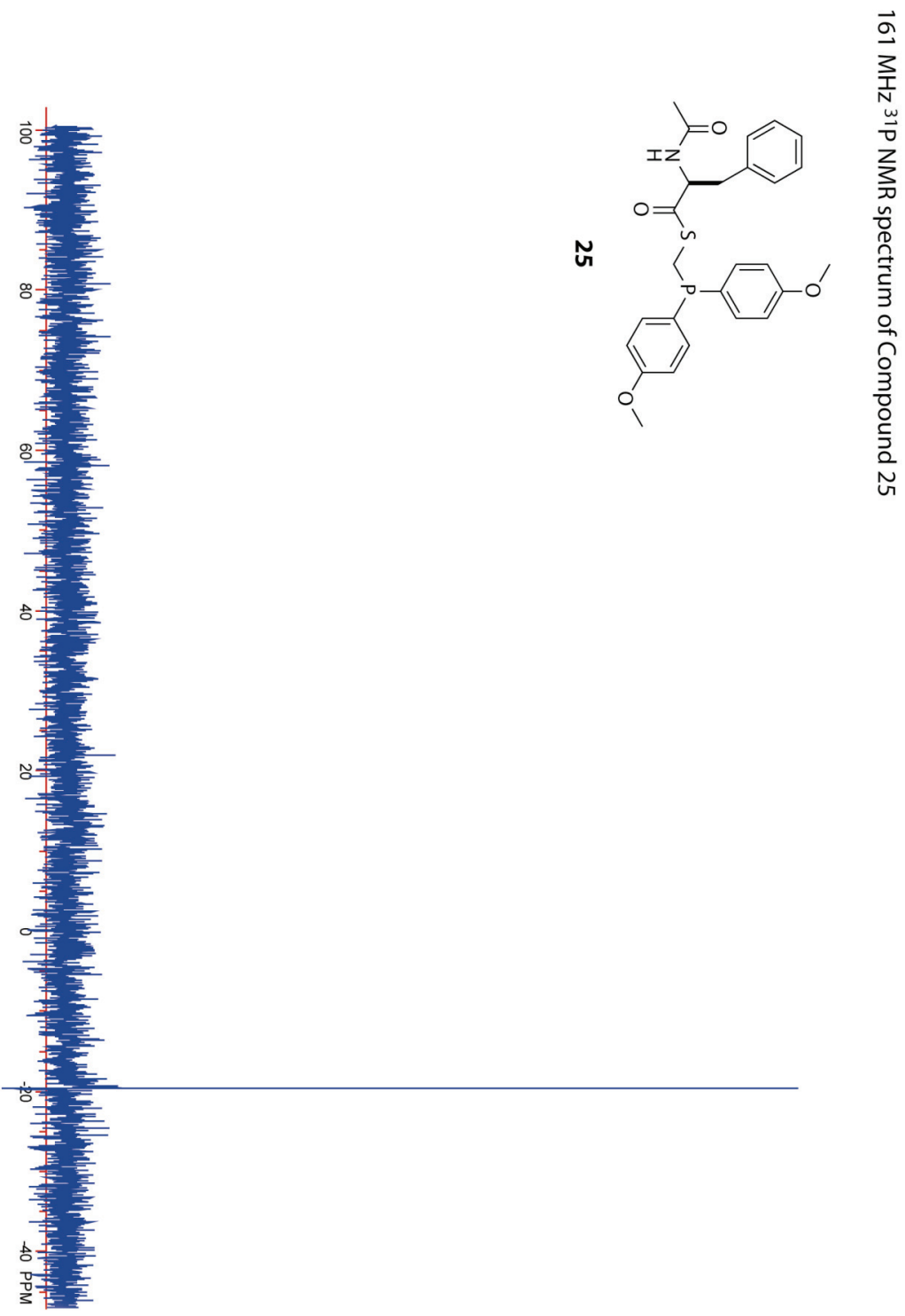


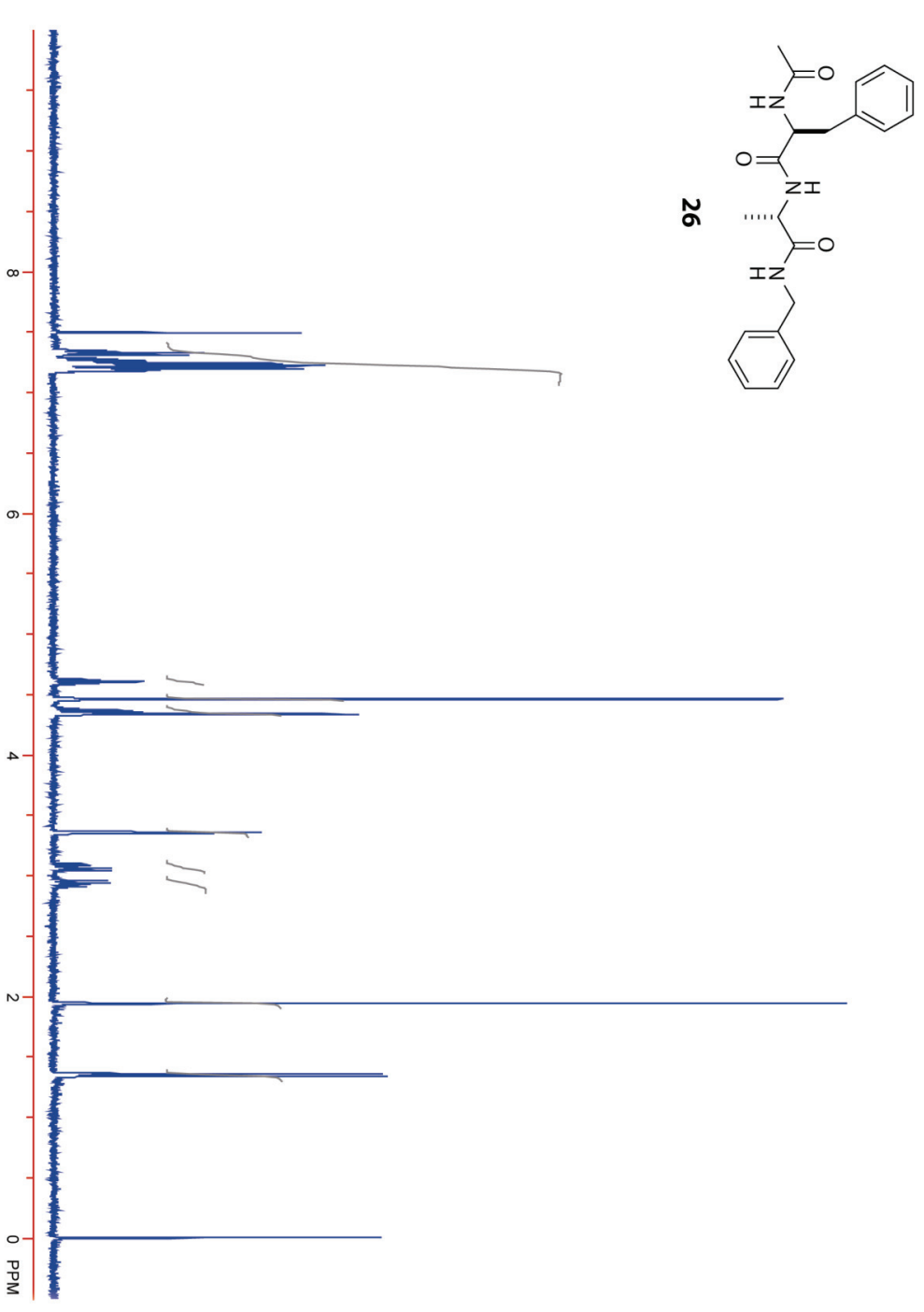

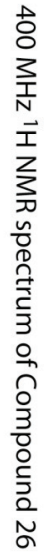



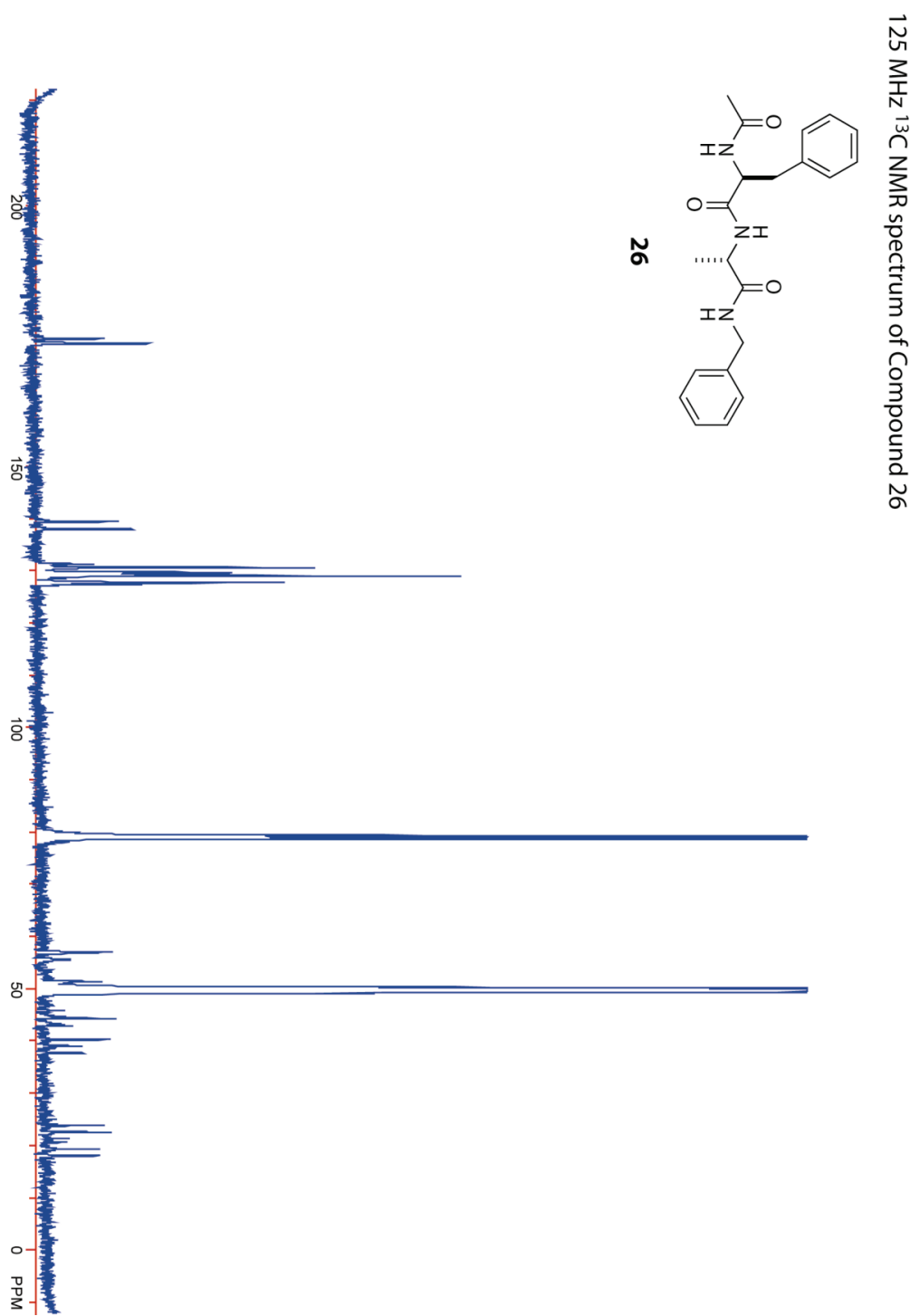
Table 1, Cl entry

Energy (Hartrees) and optimized geometry (B3L YP/6-31+G(d,p)):

$E=-695.1208559674$ ZPVE $=0.235964$<smiles>CN1C(C)(O)SC[P+]1(c1ccc(Cl)cc1)c1ccc(Cl)cc1</smiles>

\begin{tabular}{|c|c|c|c|c|c|}
\hline \multirow{2}{*}{$\begin{array}{l}\text { Center } \\
\text { Number }\end{array}$} & \multirow{2}{*}{$\begin{array}{l}\text { Atomic } \\
\text { Number }\end{array}$} & \multirow{2}{*}{$\begin{array}{c}\text { Atomic } \\
\text { Type }\end{array}$} & \multicolumn{3}{|c|}{ Coordinates (Angstroms) } \\
\hline & & & $\mathrm{X}$ & $\mathrm{Y}$ & Z \\
\hline 1 & 6 & 0 & 0.087465 & 1.897620 & 0.236664 \\
\hline 2 & 1 & 0 & 0.763455 & 1.627702 & 1.059299 \\
\hline 3 & 1 & 0 & -0.908136 & 2.053239 & 0.674989 \\
\hline 4 & 15 & 0 & -0.014978 & 0.481974 & -1.037368 \\
\hline 5 & 16 & 0 & 0.652079 & 3.279920 & -0.745653 \\
\hline 6 & 6 & 0 & 0.930322 & 4.658624 & 0.425765 \\
\hline 7 & 6 & 0 & 0.686879 & 4.398047 & 1.891132 \\
\hline 8 & 1 & 0 & -0.330365 & 4.019619 & 2.061876 \\
\hline 9 & 1 & 0 & 1.393182 & 3.648920 & 2.275219 \\
\hline 10 & 1 & 0 & 0.807687 & 5.304554 & 2.500347 \\
\hline 11 & 8 & 0 & 1.301472 & 5.742801 & 0.033235 \\
\hline 12 & 6 & 0 & -1.585797 & -0.321909 & -0.468370 \\
\hline 13 & 6 & 0 & -2.747954 & 0.361930 & -0.812976 \\
\hline 14 & 6 & 0 & -1.669177 & -1.551367 & 0.172937 \\
\hline 15 & 6 & 0 & -3.995169 & -0.175209 & -0.514242 \\
\hline 16 & 1 & 0 & -2.676151 & 1.329083 & -1.326692 \\
\hline 17 & 6 & 0 & -2.910070 & -2.103373 & 0.475414 \\
\hline 18 & 1 & 0 & -0.750994 & -2.089760 & 0.444276 \\
\hline 19 & 6 & 0 & -4.066875 & -1.408851 & 0.130129 \\
\hline 20 & 1 & 0 & -4.914044 & 0.357424 & -0.782563 \\
\hline 21 & 1 & 0 & -2.982797 & -3.073546 & 0.978812 \\
\hline 22 & 6 & 0 & 1.318758 & -0.659998 & -0.443357 \\
\hline 23 & 6 & 0 & 2.111169 & -1.153375 & -1.474149 \\
\hline 24 & 6 & 0 & 1.587007 & -1.017915 & 0.872933 \\
\hline 25 & 6 & 0 & 3.173376 & -2.008302 & -1.197002 \\
\hline 26 & 1 & 0 & 1.893962 & -0.858976 & -2.509022 \\
\hline 27 & 6 & 0 & 2.646496 & -1.870694 & 1.165360 \\
\hline 28 & 1 & 0 & 0.965953 & -0.631536 & 1.690477 \\
\hline 29 & 6 & 0 & 3.433422 & -2.361350 & 0.125301 \\
\hline 30 & 1 & 0 & 3.802686 & -2.400379 & -2.003481 \\
\hline 31 & 1 & 0 & 2.865786 & -2.157453 & 2.199600 \\
\hline 32 & 17 & 0 & 4.716987 & -3.394384 & 0.473080 \\
\hline 33 & 17 & 0 & -5.572851 & -2.069316 & 0.494492 \\
\hline
\end{tabular}


Table 1, H entry

Energy (Hartrees) and optimized geometry (B3LYP/6-31+G(d,p)):

$E=-586.9660504650 \quad Z P V E=0.254291$



\begin{tabular}{|c|c|c|c|c|c|}
\hline \multirow{2}{*}{$\begin{array}{l}\text { Center } \\
\text { Number }\end{array}$} & \multirow{2}{*}{$\begin{array}{l}\text { Atomic } \\
\text { Number }\end{array}$} & \multirow{2}{*}{$\begin{array}{c}\text { Atomic } \\
\text { Type }\end{array}$} & \multicolumn{3}{|c|}{ Coordinates (Angstroms) } \\
\hline & & & $\mathrm{x}$ & $\mathrm{Y}$ & Z \\
\hline 1 & 6 & 0 & 0.000000 & 0.000000 & 0.000000 \\
\hline 2 & 1 & 0 & 0.000000 & 0.000000 & 1.099057 \\
\hline 3 & 1 & 0 & 1.050846 & 0.000000 & -0.320794 \\
\hline 4 & 15 & 0 & -0.875090 & 1.580726 & -0.611304 \\
\hline 5 & 16 & 0 & -0.979824 & -1.317847 & -0.704786 \\
\hline 6 & 6 & 0 & -0.098723 & -2.871334 & -0.309881 \\
\hline 7 & 6 & 0 & 1.196692 & -2.773406 & 0.456627 \\
\hline 8 & 1 & 0 & 1.960185 & -2.250699 & -0.136195 \\
\hline 9 & 1 & 0 & 1.060240 & -2.217465 & 1.394381 \\
\hline 10 & 1 & 0 & 1.602632 & -3.761190 & 0.715284 \\
\hline 11 & 8 & 0 & -0.542198 & -3.944355 & -0.656656 \\
\hline 12 & 6 & 0 & 0.353147 & 2.200615 & -1.852595 \\
\hline 13 & 6 & 0 & -0.174811 & 2.380910 & -3.125341 \\
\hline 14 & 6 & 0 & 1.692526 & 2.478115 & -1.611298 \\
\hline 15 & 6 & 0 & 0.636360 & 2.840509 & -4.159290 \\
\hline 16 & 1 & 0 & -1.232701 & 2.154471 & -3.308017 \\
\hline 17 & 6 & 0 & 2.506528 & 2.938282 & -2.641952 \\
\hline 18 & 1 & 0 & 2.109152 & 2.342342 & -0.605327 \\
\hline 19 & 6 & 0 & 1.977607 & 3.119168 & -3.916440 \\
\hline 20 & 1 & 0 & 0.219469 & 2.980931 & -5.161937 \\
\hline 21 & 1 & 0 & 3.561480 & 3.159219 & -2.450038 \\
\hline 22 & 1 & 0 & 2.617599 & 3.480992 & -4.727905 \\
\hline 23 & 6 & 0 & -0.604607 & 2.724457 & 0.823624 \\
\hline 24 & 6 & 0 & -1.636570 & 3.638396 & 1.001596 \\
\hline 25 & 6 & 0 & 0.476694 & 2.724019 & 1.695813 \\
\hline 26 & 6 & 0 & -1.588378 & 4.554240 & 2.048705 \\
\hline 27 & 1 & 0 & -2.489438 & 3.629416 & 0.311472 \\
\hline 28 & 6 & 0 & 0.527908 & 3.636429 & 2.745613 \\
\hline 29 & 1 & 0 & 1.295076 & 2.005699 & 1.561890 \\
\hline 30 & 6 & 0 & -0.504700 & 4.552405 & 2.921307 \\
\hline 31 & 1 & 0 & -2.402241 & 5.273694 & 2.185997 \\
\hline 32 & 1 & 0 & 1.380740 & 3.634250 & 3.432165 \\
\hline 33 & 1 & 0 & -0.464343 & 5.270930 & 3.746574 \\
\hline
\end{tabular}


Table 1, OMe entry

Energy (Hartrees) and optimized geometry (B3LYP/6-31+G(d,p)):

$E=-710.3092017285 \quad Z P V E=0.289409$

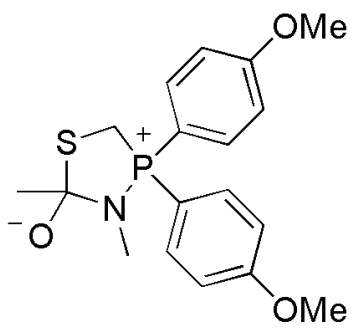

\begin{tabular}{|c|c|c|c|c|c|}
\hline \multirow{2}{*}{$\begin{array}{l}\text { Center } \\
\text { Number }\end{array}$} & \multirow{2}{*}{$\begin{array}{l}\text { Atomic } \\
\text { Number }\end{array}$} & \multirow{2}{*}{$\begin{array}{l}\text { Atomic } \\
\text { Type }\end{array}$} & \multicolumn{3}{|c|}{ Coordinates (Angstroms) } \\
\hline & & & $\mathrm{x}$ & $\mathrm{Y}$ & Z \\
\hline 1 & 6 & 0 & 0.000000 & 0.000000 & 0.000000 \\
\hline 2 & 1 & 0 & 0.000000 & 0.000000 & 1.098235 \\
\hline 3 & 1 & 0 & 1.050033 & 0.000000 & -0.324081 \\
\hline 4 & 15 & 0 & -0.838331 & 1.582446 & -0.666642 \\
\hline 5 & 16 & 0 & -0.965191 & -1.317421 & -0.725521 \\
\hline 6 & 6 & 0 & -0.293159 & -2.874031 & -0.041014 \\
\hline 7 & 6 & 0 & 0.848235 & -2.779129 & 0.940626 \\
\hline 8 & 1 & 0 & 1.694521 & -2.226749 & 0.509441 \\
\hline 9 & 1 & 0 & 0.534107 & -2.253311 & 1.852944 \\
\hline 10 & 1 & 0 & 1.220237 & -3.767614 & 1.243661 \\
\hline 11 & 8 & 0 & -0.752206 & -3.948062 & -0.364899 \\
\hline 12 & 6 & 0 & 0.669532 & 2.615682 & -0.950232 \\
\hline 13 & 6 & 0 & 1.406436 & 2.280533 & -2.081831 \\
\hline 14 & 6 & 0 & 1.047618 & 3.710225 & -0.183468 \\
\hline 15 & 6 & 0 & 2.519336 & 3.024724 & -2.448594 \\
\hline 16 & 1 & 0 & 1.100016 & 1.419821 & -2.689762 \\
\hline 17 & 6 & 0 & 2.155679 & 4.470866 & -0.534594 \\
\hline 18 & 1 & 0 & 0.464952 & 3.975713 & 0.709267 \\
\hline 19 & 6 & 0 & 2.903636 & 4.127597 & -1.670357 \\
\hline 20 & 1 & 0 & 3.088314 & 2.745309 & -3.343250 \\
\hline 21 & 1 & 0 & 2.437154 & 5.333025 & 0.081571 \\
\hline 22 & 6 & 0 & -1.594184 & 2.288450 & 0.865402 \\
\hline 23 & 6 & 0 & -2.891515 & 2.750551 & 0.672417 \\
\hline 24 & 6 & 0 & -1.014975 & 2.368009 & 2.126140 \\
\hline 25 & 6 & 0 & -3.613985 & 3.293701 & 1.726695 \\
\hline 26 & 1 & 0 & -3.343207 & 2.677754 & -0.325257 \\
\hline 27 & 6 & 0 & -1.720798 & 2.905544 & 3.194861 \\
\hline 28 & 1 & 0 & 0.007961 & 2.005698 & 2.287490 \\
\hline 29 & 6 & 0 & -3.027642 & 3.377523 & 2.998731 \\
\hline 30 & 1 & 0 & -4.636003 & 3.652551 & 1.556295 \\
\hline 31 & 1 & 0 & -1.249420 & 2.959643 & 4.183326 \\
\hline 32 & 7 & 0 & 4.104616 & 4.835042 & -1.976905 \\
\hline 33 & 1 & 0 & 4.319942 & 4.795675 & -2.948179 \\
\hline 34 & 1 & 0 & 4.073850 & 5.775417 & -1.651285 \\
\hline 35 & 7 & 0 & -3.718716 & 4.028739 & 4.063216 \\
\hline 36 & 1 & 0 & -4.706724 & 3.967446 & 3.957389 \\
\hline 37 & 1 & 0 & -3.427958 & 3.694218 & 4.954648 \\
\hline
\end{tabular}


Table 2, $\mathrm{H}_{2} \mathrm{O}$ entry

Energy (Hartrees) and optimized geometry $\left(\mathrm{B} 3 \mathrm{LYP} / 6-31+\mathrm{G}(\mathrm{d}, \mathrm{p}), \mathrm{scrf}=\left(\mathrm{cpcm}\right.\right.$, solvent $\left.\left.=\mathrm{H}_{2} \mathrm{O}\right)\right)$ : $E=-780.6118320298 \quad Z P V E=0.297677$

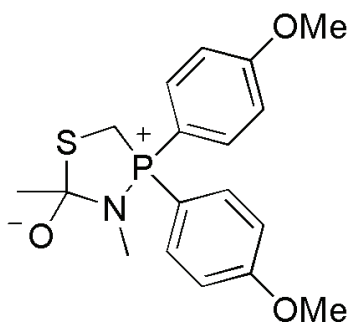

\begin{tabular}{|c|c|c|c|c|c|}
\hline \multirow{2}{*}{$\begin{array}{l}\text { Center } \\
\text { Number }\end{array}$} & \multirow{2}{*}{$\begin{array}{l}\text { Atomic } \\
\text { Number }\end{array}$} & \multirow{2}{*}{$\begin{array}{c}\text { Atomic } \\
\text { Type }\end{array}$} & \multicolumn{3}{|c|}{ Coordinates (Angstroms) } \\
\hline & & & $\mathrm{X}$ & Y & $\mathrm{Z}$ \\
\hline--- & & & 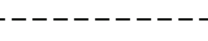 & -------- & ---------- \\
\hline 1 & 7 & 0 & 0.000000 & 0.000000 & 0.000000 \\
\hline 2 & 6 & 0 & 0.000000 & 0.000000 & 1.577141 \\
\hline 3 & 16 & 0 & 2.215100 & 0.000000 & 1.772724 \\
\hline 4 & 6 & 0 & 2.598875 & -1.100987 & 0.422063 \\
\hline 5 & 8 & 0 & -0.530826 & -1.007783 & 2.052438 \\
\hline 6 & 6 & 0 & 3.024196 & 2.291200 & -3.737407 \\
\hline 7 & 6 & 0 & 3.002645 & 2.528966 & -2.367099 \\
\hline 8 & 6 & 0 & 2.474782 & 1.573851 & -1.503331 \\
\hline 9 & 6 & 0 & 1.963909 & 0.378442 & -2.007599 \\
\hline 10 & 6 & 0 & 1.990809 & 0.144343 & -3.378997 \\
\hline 11 & 6 & 0 & 2.519710 & 1.096285 & -4.243379 \\
\hline 12 & 6 & 0 & -1.329047 & -0.091959 & -0.617294 \\
\hline 13 & 6 & 0 & -0.220186 & 1.402598 & 2.126633 \\
\hline 14 & 1 & 0 & 3.605171 & -0.905066 & 0.020425 \\
\hline 15 & 1 & 0 & 2.595407 & -2.170722 & 0.706857 \\
\hline 16 & 1 & 0 & 3.439116 & 3.042054 & -4.418595 \\
\hline 17 & 1 & 0 & 3.401472 & 3.466258 & -1.963809 \\
\hline 18 & 1 & 0 & 2.464421 & 1.747409 & -0.410403 \\
\hline 19 & 1 & 0 & 1.592880 & -0.796874 & -3.782128 \\
\hline 20 & 1 & 0 & 2.538618 & 0.905416 & -5.321602 \\
\hline 21 & 1 & 0 & -1.241042 & -0.170710 & -1.714729 \\
\hline 22 & 1 & 0 & -1.914119 & -0.950103 & -0.253286 \\
\hline 23 & 1 & 0 & -1.878301 & 0.826778 & -0.370917 \\
\hline 24 & 1 & 0 & -0.049832 & 1.414639 & 3.211297 \\
\hline 25 & 1 & 0 & 0.449791 & 2.145171 & 1.673688 \\
\hline 26 & 1 & 0 & -1.254357 & 1.727516 & 1.946610 \\
\hline 27 & 15 & 0 & 1.322679 & -0.777682 & -0.813530 \\
\hline 28 & 6 & 0 & 0.846679 & -2.294516 & -1.645043 \\
\hline 29 & 6 & 0 & 1.464330 & -3.485688 & -1.272231 \\
\hline 30 & 6 & 0 & -0.138669 & -2.322545 & -2.629407 \\
\hline 31 & 6 & 0 & 1.124328 & -4.684428 & -1.890260 \\
\hline 32 & 1 & 0 & 2.215642 & -3.471994 & -0.463898 \\
\hline 33 & 6 & 0 & -0.482760 & -3.516870 & -3.253015 \\
\hline 34 & 1 & 0 & -0.670222 & -1.391118 & -2.888299 \\
\hline 35 & 6 & 0 & 0.153401 & -4.699450 & -2.886854 \\
\hline 36 & 1 & 0 & 1.615917 & -5.615142 & -1.586575 \\
\hline 37 & 1 & 0 & -1.259043 & -3.527036 & -4.025706 \\
\hline 38 & 1 & 0 & -0.116466 & -5.641702 & -3.376230 \\
\hline
\end{tabular}


Table 2, THF entry

Energy (Hartrees) and optimized geometry $(B 3 \mathrm{LYP} / 6-31+\mathrm{G}(\mathrm{d}, \mathrm{p}), \mathrm{scrf}=(\mathrm{cpcm}$, solvent $=\mathrm{THF}))$ : $E=-780.6118320298 \quad Z P V E=0.297677$

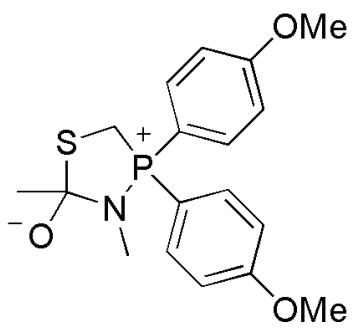

\begin{tabular}{|c|c|c|c|c|c|}
\hline \multirow{2}{*}{$\begin{array}{l}\text { Center } \\
\text { Number }\end{array}$} & \multirow{2}{*}{$\begin{array}{l}\text { Atomic } \\
\text { Number }\end{array}$} & \multirow{2}{*}{$\begin{array}{l}\text { Atomic } \\
\text { Type }\end{array}$} & \multicolumn{3}{|c|}{ Coordinates (Angstroms) } \\
\hline & & & $\mathrm{x}$ & $\mathrm{Y}$ & Z \\
\hline 1 & 7 & 0 & 0.000000 & 0.000000 & 0.000000 \\
\hline 2 & 6 & 0 & 0.000000 & 0.000000 & 1.577141 \\
\hline 3 & 16 & 0 & 2.215100 & 0.000000 & 1.772724 \\
\hline 4 & 6 & 0 & 2.598875 & -1.100987 & 0.422063 \\
\hline 5 & 8 & 0 & -0.530826 & -1.007783 & 2.052438 \\
\hline 6 & 6 & 0 & 3.024196 & 2.291200 & -3.737407 \\
\hline 7 & 6 & 0 & 3.002645 & 2.528966 & -2.367099 \\
\hline 8 & 6 & 0 & 2.474782 & 1.573851 & -1.503331 \\
\hline 9 & 6 & 0 & 1.963909 & 0.378442 & -2.007599 \\
\hline 10 & 6 & 0 & 1.990809 & 0.144343 & -3.378997 \\
\hline 11 & 6 & 0 & 2.519710 & 1.096285 & -4.243379 \\
\hline 12 & 6 & 0 & -1.329047 & -0.091959 & -0.617294 \\
\hline 13 & 6 & 0 & -0.220186 & 1.402598 & 2.126633 \\
\hline 14 & 1 & 0 & 3.605171 & -0.905066 & 0.020425 \\
\hline 15 & 1 & 0 & 2.595407 & -2.170722 & 0.706857 \\
\hline 16 & 1 & 0 & 3.439116 & 3.042054 & -4.418595 \\
\hline 17 & 1 & 0 & 3.401472 & 3.466258 & -1.963809 \\
\hline 18 & 1 & 0 & 2.464421 & 1.747409 & -0.410403 \\
\hline 19 & 1 & 0 & 1.592880 & -0.796874 & -3.782128 \\
\hline 20 & 1 & 0 & 2.538618 & 0.905416 & -5.321602 \\
\hline 21 & 1 & 0 & -1.241042 & -0.170710 & -1.714729 \\
\hline 22 & 1 & 0 & -1.914119 & -0.950103 & -0.253286 \\
\hline 23 & 1 & 0 & -1.878301 & 0.826778 & -0.370917 \\
\hline 24 & 1 & 0 & -0.049832 & 1.414639 & 3.211297 \\
\hline 25 & 1 & 0 & 0.449791 & 2.145171 & 1.673688 \\
\hline 26 & 1 & 0 & -1.254357 & 1.727516 & 1.946610 \\
\hline 27 & 15 & 0 & 1.322679 & -0.777682 & -0.813530 \\
\hline 28 & 6 & 0 & 0.846679 & -2.294516 & -1.645043 \\
\hline 29 & 6 & 0 & 1.464330 & -3.485688 & -1.272231 \\
\hline 30 & 6 & 0 & -0.138669 & -2.322545 & -2.629407 \\
\hline 31 & 6 & 0 & 1.124328 & -4.684428 & -1.890260 \\
\hline 32 & 1 & 0 & 2.215642 & -3.471994 & -0.463898 \\
\hline 33 & 6 & 0 & -0.482760 & -3.516870 & -3.253015 \\
\hline 34 & 1 & 0 & -0.670222 & -1.391118 & -2.888299 \\
\hline 35 & 6 & 0 & 0.153401 & -4.699450 & -2.886854 \\
\hline 36 & 1 & 0 & 1.615917 & -5.615142 & -1.586575 \\
\hline 37 & 1 & 0 & -1.259043 & -3.527036 & -4.025706 \\
\hline 38 & 1 & 0 & -0.116466 & -5.641702 & -3.376230 \\
\hline
\end{tabular}

